\title{
PRESCRIBED MEAN CURVATURE EQUATION ON THE UNIT BALL IN THE PRESENCE OF REFLECTION OR ROTATION SYMMETRY
}

\author{
PAK TUNG HO
}

\begin{abstract}
Using the flow method, we prove some existence results for the problem of prescribing the mean curvature on the unit ball. More precisely, we prove that there exists a conformal metric on the unit ball such that its mean curvature is $f$, when $f$ possesses certain reflection or rotation symmetry.
\end{abstract}

\section{INTRODUCTION}

The problem of prescribing scalar curvature on a closed manifold has been studied extensively for last few decades. More precisely, let $(M, g)$ be an $n$-dimensional compact smooth Riemannian manifold without boundary. Given a smooth function $f$ on $M$, can we find a metric conformal to $g$ such that its scalar curvature is $f$ ? This has been studied in $3,5,14,28,33,34,38,43$. When $(M, g)$ is the $n$ dimensional standard sphere $S^{n}$, it is called the Nirenberg's problem and has been studied extensively. See [4, 7, 9, 10, 11, 15, 25, 26, 39, 44, 45] and the references therein. In particular, Chang-Yang obtained in [9] a perturbation theorem which asserts that there exists a conformal metric whose scalar curvature is equal to $f$, provided that the degree condition holds for $f$ which is a positive Morse function and is sufficiently closed to $n(n-1)$ in $C^{0}$ norm. See also [18, 19, 29, 30, 31, 32, 37, for related results of prescribing the Webster scalar curvature on CR manifolds.

A geometric flow has been introduced to study the Nirenberg's problem by Struwe in 42 for $n=2$, and has been generalized to $n \geq 3$ by Chen-Xu in [16]. More precisely, the scalar curvature flow is defined as

$$
\frac{\partial}{\partial t} u=\frac{n-2}{4}\left(\alpha f-R_{\tilde{g}}\right) u,
$$

where $R_{\tilde{g}}$ is the scalar curvature of $\tilde{g}=u^{\frac{4}{n-2}} g$ and $\alpha$ is a constant chosen to preserve the volume along the flow. Using the scalar curvature flow, Chen-Xu [16] was able to prove Chang-Yang's result with the quantitative bound on $\|f-n(n-1)\|_{C^{0}\left(S^{n}\right)}$.

Again using the scalar curvature flow, Leung-Zhou [35] proved an existence result for prescribing scalar curvature with symmetry. To describe their result, we have the following:

Assumption 1.1. $f$ is symmetric under a mirror reflection upon a hyperplane $\mathcal{H} \subset \mathbb{R}^{n+1}$ passing through the origin.

Date: 2nd January, 2017.

2000 Mathematics Subject Classification. Primary 53C44, 53A30; Secondary 35J93, 35B44.

Key words and phrases. mean curvature; unit ball; Nirenberg's problem. 
Under Assumption 1.1, without loss of generality, we may assume that $\mathcal{H}$ is the hyperplane perpendicular to the $x_{1}$-axis. Then the symmetry can be expressed as

$$
f\left(\gamma_{m}(x)\right)=f(x)
$$

where $\gamma_{m}: S^{n} \rightarrow S^{n}$ is given by

$$
\gamma_{m}\left(x_{1}, x_{2}, \ldots, x_{n+1}\right)=\left(-x_{1}, x_{2}, \ldots, x_{n+1}\right) \quad \text { for } x=\left(x_{1}, x_{2}, \ldots, x_{n+1}\right) \in S^{n} .
$$

Then

$$
\mathcal{F}=\mathcal{H} \cap S^{n}=\left\{\left(0, x_{2}, \ldots, x_{n+1}\right) \in S^{n}\right\}
$$

is the fixed point set.

Assumption 1.2. $f$ is invariant under a rotation $\gamma_{\theta}$ of angle $\theta=\pi / k$ with the rotation axis being a straight line in $\mathbb{R}^{n+1}$ passing through the origin. Here $k>1$ is an integer.

Under Assumption 1.2, without loss of generality, we may assume that the rotation axis is the $x_{n+1}$-axis. In this case, $\mathcal{F}=\{N, S\}$ is the fixed point set, where $N=(0, \ldots, 0,1)$ is the north pole and $S=(0, \ldots, 0,-1)$ is the south pole.

With these assumptions, we can state the result of Leung-Zhou in 35].

Theorem 1.1 (Leung-Zhou [35]). Suppose that $f$ is a positive smooth function on $S^{n}$ satisfying Assumption 1.1 or [1.2, Assume that

$$
x_{m} \in \mathcal{F} \text { with } f\left(x_{m}\right)=\max _{\mathcal{F}} f \Rightarrow \Delta_{S^{n}} f\left(x_{m}\right)>0
$$

where $\Delta_{S^{n}}$ is the Laplacian of the standard metric of $S^{n}$, and

$$
\max _{S^{n}} f<2^{\frac{2}{n-2}}\left(\max _{\mathcal{F}} f\right) .
$$

Then $f$ can be realized as the scalar curvature of some metric conformal to the standard metric of $S^{n}$.

Note that existence results for prescribing scalar curvature with symmetry were obtained earlier by Moser [36] and by Escobar-Schoen [24].

The problem of prescribing the scalar curvature or the mean curvature has been studied on manifolds with boundary. See [12, 13, 20, 21, 27, 40, 47] for example. In this paper, we consider the following problem of prescribing the mean curvature on the unit ball, which is a natural analogy of the prescribing scalar curvature problem: Let $B^{n+1}$ be the $(n+1)$-dimensional unit ball equipped with the flat metric $g_{0}$, i.e.

$$
B^{n+1}=\left\{\left(x_{1}, \ldots, x_{n}, x_{n+1}\right) \in \mathbb{R}^{n+1}: \sum_{i=1}^{n+1} x_{i}^{2} \leq 1\right\} .
$$

The boundary of $B^{n+1}$ is the $n$-dimensional unit sphere $S^{n}$, i.e.

$$
\partial B^{n+1}=S^{n}=\left\{\left(x_{1}, \ldots, x_{n}, x_{n+1}\right) \in \mathbb{R}^{n+1}: \sum_{i=1}^{n+1} x_{i}^{2}=1\right\} .
$$

The mean curvature $H_{g_{0}}$ of $S^{n}$ with respect to $g_{0}$ is equal to 1 , i.e. $H_{g_{0}}=1$. On the other hand, the metric induced by $g_{0}$ on $S^{n}$ is the standard metric $g_{S^{n}}$ of $S^{n}$. We study the following problem: given a smooth function $f$ on $S^{n}$, find a metric conformal to $g_{0}$ such that it is flat in the interior of $B^{n+1}$ and its mean curvature 
is equal to $f$ on $\partial B^{n+1}=S^{n}$. The problem is equivalent to finding a positive harmonic function $u$ in the ball with nonlinear boundary condition:

$$
\begin{aligned}
\Delta_{g_{0}} u & =0 \quad \text { in } B^{n+1}, \\
\frac{2}{n-1} \frac{\partial u}{\partial \nu_{g_{0}}}+u & =f u^{\frac{n+1}{n-1}} \quad \text { on } S^{n} .
\end{aligned}
$$

Here, $\Delta_{g_{0}}$ is the Laplacian of $g_{0}$ and $\frac{\partial}{\partial \nu_{g_{0}}}$ is the outward normal derivative of $g_{0}$. See 1, 2, 6, 17, 20, 21, 23, 40, 41 and references therein for the results related to this problem. In particular, Chang-Xu-Yang proved in [8 that (1.1) has a solution when $f$ is a positive Morse function satisfying the degree condition and being sufficiently closed to 1 in $C^{0}$ norm. By the method of geometric flow, Xu-Zhang [46] proved Chang-Xu-Yang's result with the quantitative bound on $\|f-1\|_{C^{0}\left(S^{n}\right)}$.

Inspired by the result of Leung-Zhou [35, we study the problem of prescribing the mean curvature on the unit ball with symmetry. We prove the following:

Theorem 1.2. Suppose that $f$ is a positive smooth function on $S^{n}$ satisfying Assumption 1.1 or [1.2. Assume that

$$
x_{m} \in \mathcal{F} \text { with } f\left(x_{m}\right)=\max _{\mathcal{F}} f \Rightarrow \Delta_{S^{n}} f\left(x_{m}\right)>0
$$

where $\Delta_{S^{n}}$ is the Laplacian of the standard metric of $S^{n}$, and

$$
\max _{S^{n}} f<2^{\frac{1}{n-1}}\left(\max _{\mathcal{F}} f\right) .
$$

Then $f$ can be realized as the mean curvature of some conformal metric on the unit ball $B^{n+1}$.

We remark that Escobar has also studied in 21 the existence of a positive solution to (1.1) when $f$ has symmetry. The proof of Theorem 1.2 follows the arguments of Leung-Zhou in [35. We also remark that we have used the arguments of Leung-Zhou to study the problem of prescribing Webster scalar curvature on the CR sphere with symmetry. See [30].

Acknowledgements. Part of the work was done while the author was visiting Princeton University in 2016. He thanks Prof. Paul C. Yang for the invitation and is grateful to Princeton University for the kind hospitality. The author was supported by the National Research Foundation of Korea (NRF) grant funded by the Korea government (MEST) (No.201631023.01).

\section{The FLOW AND ITS PROPERTIES}

Let $B^{n+1}$ be the $(n+1)$-dimensional unit ball equipped with the flat metric $g_{0}$. Then the $n$-dimensional sphere $S^{n}=\partial B^{n+1}$ is the boundary of $B^{n+1}$. Let $f$ be a positive smooth function defined on $S^{n}$. Given a smooth function $u_{0}$ in $B^{n+1}$, we consider the flow

$$
u_{t}=\frac{n-1}{4}\left(\alpha f-H_{g}\right) u \quad \text { on } S^{n}
$$

for $t \geq 0$ with the initial condition

$$
\left.u\right|_{t=0}=u_{0},
$$


where

$$
H_{g}=u^{-\frac{n+1}{n-1}}\left(\frac{2}{n-1} \frac{\partial u}{\partial \nu_{g_{0}}}+u\right) \quad \text { on } S^{n}
$$

is the mean curvature of the conformal metric $g=u^{\frac{4}{n-1}} g_{0}$, and

$$
\Delta_{g_{0}} u=0 \text { in } B^{n+1} \text {. }
$$

Here, $\Delta_{g_{0}}$ is the Laplacian of $g_{0}$ and $\frac{\partial}{\partial \nu_{g_{0}}}$ is the outward normal derivative of $g_{0}$. Also, $\alpha=\alpha(t)$ is defined as

$$
\alpha=\left(f_{S^{n}} H_{g} u^{\frac{2 n}{n-1}} d \mu\right) /\left(f_{S^{n}} f u^{\frac{2 n}{n-1}} d \mu\right),
$$

where $d \mu$ is the volume form of the standard metric $g_{S^{n}}$ on $S^{n}$ and

$$
f_{S^{n}} \varphi d \mu=\frac{1}{\omega_{n}} \int_{S^{n}} \varphi d \mu \text { for all } \varphi \in C^{\infty}\left(S^{n}\right),
$$

where $\omega_{n}=\int_{S^{n}} d \mu$ is the volume of $S^{n}$ with respect to $g_{S^{n}}$.

We define the functionals

$$
E[u]=f_{S^{n}}\left(\frac{2}{n-2} \frac{\partial u}{\partial \nu_{g_{0}}}+u\right) u d \mu=f_{S^{n}} H_{g} u^{\frac{2 n}{n-1}} d \mu
$$

and

$$
E_{f}[u]=E[u] /\left(f_{S^{n}} f u^{\frac{2 n}{n-1}} d \mu\right)^{\frac{n-1}{n}} .
$$

It follows from Lemma 2.2 in [46] that

$$
\frac{d}{d t} E_{f}[u] \leq 0
$$

along the flow (2.1). In particular, we have

$$
E_{f}[u] \leq E_{f}\left[u_{0}\right] \text { for all } t \geq 0 .
$$

It follows from section 2.3 in [46] that (2.1)-(2.3) has a unique solution $u(x, t)$ on $B^{n+1} \times[0, \infty)$ such that, given $T>0$, there exists a constant $C=C(T)$ such that

$$
C^{-1} \leq u(x, t) \leq C \text { for all }(x, t) \in B^{n+1} \times[0, T] .
$$

Lemma 2.1. Given an isometry $\gamma:\left(S^{n}, g_{S^{n}}\right) \rightarrow\left(S^{n}, g_{S^{n}}\right)$, we assume that

$$
f(\gamma(x))=f(x) \text { and } u_{0}(\gamma(x))=u_{0}(x) \text { for all } x \in S^{n} .
$$

Let $u(x, t)$ be the solution of (2.1)-(2.3) with initial data $u_{0}$. Then

$$
u(\gamma(x), t)=u(x, t) \quad \text { for all }(x, t) \in S^{n} \times[0, \infty) .
$$

Proof. Since $\gamma:\left(S^{n}, g_{S^{n}}\right) \rightarrow\left(S^{n}, g_{S^{n}}\right)$ is isometry, for any $\varphi \in C^{\infty}\left(B^{2 n+1}\right)$, we have

$$
\left(\frac{\partial(\varphi \circ \gamma)}{\partial \nu_{g_{0}}}\right)(x)=\left(\frac{\partial \varphi}{\partial \nu_{g_{0}}}\right)(\gamma(x)) \text { for all } x \in S^{n} .
$$

Since the mean curvature of $g(x, t)=u(x, t)^{\frac{4}{n-1}} g_{0}$ satisfies

$$
H_{g(x, t)}(x)=u(x, t)^{-\frac{n+1}{n-1}}\left(\frac{2}{n-1} \frac{\partial}{\partial \nu_{g_{0}}} u(x, t)+u(x, t)\right) \text { for } x \in S^{n},
$$


it follows from (2.10) and (2.11) that

$$
H_{g(x, t)}(\gamma(x))=H_{g(\gamma(x), t)}(x) \text { for all } x \in S^{n} .
$$

By (2.1)-(2.4), the flow can be written as

$$
\begin{aligned}
\Delta_{g_{0}} u & =0 \quad \text { in } B^{n+1}, \\
u_{t} & =-\frac{n-1}{4} u^{-\frac{2}{n-1}}\left(\frac{2}{n-1} \frac{\partial u}{\partial \nu_{g_{0}}}+u\right)+\frac{n-1}{4}\left(\frac{\int_{S^{n}} H_{g} u^{\frac{2 n}{n-1}} d \mu}{\int_{S^{n}} f u^{\frac{2 n}{n-1}} d \mu}\right) f u \text { on } S^{n} .
\end{aligned}
$$

From (2.9), (2.11) and (2.12), we know that $u(\gamma(x), t)$ is also a solution of (2.13) with initial value $u_{0}$. Now the assertion (2.10) follows from the uniqueness result stated in Lemma 4.8 in [12.

For $r>0$ and $x_{0} \in S^{n}$, set $B_{r}^{+}\left(x_{0}\right)=\left\{x \in B^{n+1}: d_{g_{0}}\left(x, x_{0}\right)<r\right\}$ and $\partial^{\prime} B_{r}^{+}\left(x_{0}\right)=\partial B_{r}^{+}\left(x_{0}\right) \cap S^{n}$. The following lemma was proved in [46]: (see section 4.2 in [46])

Lemma 2.2. Let $u(t)$ be the solution of the flow (2.1). Let $\left(t_{k}\right)_{k=1}^{\infty}$ be any time sequence with $t_{k} \rightarrow \infty$ as $k \rightarrow \infty$. Consider the sequence $u_{k}:=u\left(t_{k}\right)$ and corresponding metrics $g_{k}=u\left(t_{k}\right)^{\frac{4}{n-1}} g_{0}$. Then, up to a subsequence, either

(i) the sequence $u_{k}$ is uniformly bounded in $W^{1, p}\left(S^{n}, g_{S^{n}}\right) \hookrightarrow L^{\infty}\left(S^{n}\right)$ for some $p>n$; or

(ii) there exists a subsequence of $u_{k}$ and finitely many points $x_{1}, \ldots, x_{L} \in S^{n}$ such that for any $r>0$ and any $l \in\{1, \ldots, L\}$ there holds

$$
\liminf _{k \rightarrow \infty}\left(\omega_{n}^{-1} \int_{\partial^{\prime} B_{r}\left(x_{l}\right)}\left|H_{g_{k}}\right|^{n} d \mu_{g_{k}}\right)^{\frac{1}{n}} \geq 1 .
$$

In addition, if the alternative (ii) occurs, the sequence $u_{k}$ is also uniformly bounded in $L^{p}$ on any compact subset of $\left(S^{n} \backslash\left\{x_{l}, \ldots, x_{L}\right\}, g_{S^{n}}\right)$.

Lemma 2.3. Suppose that Lemma 2.2(ii) occurs. For a point $x_{0} \in S^{n}$ with

$$
\max _{S^{n}} f<2^{\frac{1}{n-1}} f\left(x_{0}\right),
$$

suppose that the initial data $u_{0}$ satisfies

$$
E_{f}\left[u_{0}\right] \leq \frac{1}{f\left(x_{0}\right)^{\frac{n-1}{n}}}+\epsilon .
$$

Then for $\epsilon>0$ small enough, we have $L=1$.

Proof. Let $x_{1}, \ldots, x_{L} \in S^{n}$ be the blow-up points. If $L>2$, let

$$
r<\min _{1 \leq i<j \leq L} d_{g_{0}}\left(x_{i}, x_{j}\right)
$$


For any $\epsilon>0$, there exists $k$ being sufficiently large such that

$$
\begin{aligned}
L-\epsilon & \leq \sum_{l=1}^{L}\left(\omega_{n}^{-1} \int_{\partial^{\prime} B_{r}\left(x_{l}\right)}\left|H_{g_{k}}\right|^{n} d \mu_{g_{k}}\right)^{\frac{1}{n}} \\
& \leq L^{1-\frac{1}{n}}\left(\omega_{n}^{-1} \sum_{l=1}^{L} \int_{\partial^{\prime} B_{r}\left(x_{l}\right)}\left|H_{g_{k}}\right|^{n} d \mu_{g_{k}}\right)^{\frac{1}{n}} \\
& \leq L^{1-\frac{1}{n}}\left(f_{S^{n}}\left|H_{g_{k}}\right|^{n} d \mu_{g_{k}}\right)^{\frac{1}{n}} \\
& \leq L^{1-\frac{1}{n}} \alpha\left(t_{k}\right)\left(f_{S^{n}} f^{n} d \mu_{g_{k}}\right)^{\frac{1}{n}}+L^{1-\frac{1}{n}}\left(f_{S^{n}}\left|\alpha\left(t_{k}\right) f-H_{g_{k}}\right|^{n} d \mu_{g_{k}}\right)^{\frac{1}{n}},
\end{aligned}
$$

where the first inequality follows from Hölder's inequality, the second inequality follows from (2.14), and the third inequality follows from (2.17). By Lemma 3.2 in [46], for any $p \geq 2$, we have

$$
\int_{S^{n}}\left|\alpha(t) f-H_{g}\right|^{p} d \mu_{g} \rightarrow 0 \quad \text { as } t \rightarrow \infty .
$$

In particular,

$$
\int_{S^{n}}\left|\alpha\left(t_{k}\right)-H_{g_{k}}\right|^{n} d \mu_{g_{k}} \rightarrow 0 \quad \text { as } k \rightarrow \infty .
$$

On the other hand, by (2.4)-(2.6), we have

$$
\alpha=\left(f_{S^{n}} H_{g} u^{\frac{2 n}{n-1}} d \mu\right) /\left(f_{S^{n}} f u^{\frac{2 n}{n-1}} d \mu\right)=E_{f}[u] /\left(f_{S^{n}} f u^{\frac{2 n}{n-1}} d \mu\right)^{\frac{1}{n}} .
$$

Combining (2.18)-(2.20), we obtain

$$
\begin{aligned}
L-\epsilon & \leq L^{1-\frac{1}{n}} E_{f}\left[u_{k}\right]\left(f_{S^{n}} f^{n} d \mu_{g_{k}}\right)^{\frac{1}{n}} /\left(f_{S^{n}} f d \mu_{g_{k}}\right)^{\frac{1}{n}}+o(1) \\
& \leq L^{1-\frac{1}{n}} E_{f}\left[u_{k}\right]\left(\max _{S^{n}} f\right)^{\frac{n-1}{n}}+o(1) \\
& \leq L^{1-\frac{1}{n}}\left[\left(\frac{\max _{S^{n}} f}{f\left(x_{0}\right)}\right)^{\frac{n-1}{n}}+O(\epsilon)\right]+o(1) \\
& \leq L^{1-\frac{1}{n}}\left(2^{\frac{1}{n}}+O(\epsilon)\right)+o(1),
\end{aligned}
$$

where we have used (2.16) in the second last inequality, and (2.15) in the last inequality. This implies that $L=1$ when $\epsilon>0$ is small enough.

We have the following lemma regarding the blow-up point. Its proof can be found in 46.

Lemma 2.4. Under the assumptions of Lemma 2.2(ii) and 2.3, there is a point $Q \in S^{n}$ such that the following statements hold:

(a) As $k \rightarrow \infty$, the metrics $g_{k}$ concentrate at $Q$ in the sense described by (ii) in Lemma 4.2 in 46]. As a consequence, for any positive number $\rho, \max _{\partial^{\prime} B_{\rho}(Q)} u_{k}$ cannot be uniformly bounded from above for all $k \gg 1$, 
(b) $Q$ is a critical point of $f$,

(c) $\Delta_{S^{n}} f(Q) \leq 0$, and

(d) $\lim _{k \rightarrow \infty} E_{f}\left[u_{k}\right]=\frac{1}{f(Q)^{\frac{n-1}{n}}}$.

\section{Proof of Theorem 1.2}

As before, the south pole is denoted by $S=(0, \ldots, 0,-1) \in S^{n}$. Let

$$
\mathbb{R}_{+}^{n+1}=\left\{\left(z_{1}, \ldots, z_{n}, z_{n+1}\right) \in \mathbb{R}^{n+1}: z_{n+1} \geq 0\right\}
$$

be the $(n+1)$-dimensional upper half Euclidean space equipped with the Euclidean metric $g_{E}$. Consider the following map $\Sigma: B^{n+1} \backslash\{S\} \rightarrow \mathbb{R}_{+}^{n+1}$ given by

$$
\Sigma\left(x_{1}, \ldots, x_{n}, x_{n+1}\right)=\left(\frac{4 \bar{x}}{|\bar{x}|^{2}+\left(1+x_{n+1}\right)^{2}}, \frac{\left.2\left(1-|\bar{x}|^{2}-x_{n+1}^{2}\right)\right)}{|\bar{x}|^{2}+\left(1+x_{n+1}\right)^{2}}\right)
$$

where $\bar{x}=\left(x_{1}, \ldots, x_{n}\right)$. Note that

$$
\Sigma(x)=\left(\frac{2 \bar{x}}{1+x_{n+1}}, 0\right) \text { for } x \in S^{n} \backslash\{S\} .
$$

Also, $\Sigma^{-1}: \mathbb{R}_{+}^{n+1} \rightarrow B^{n+1} \backslash\{S\}$ is given by

$$
\Sigma^{-1}\left(\bar{z}, z_{n+1}\right)=\left(\frac{4 \bar{z}}{|\bar{z}|^{2}+\left(2+z_{n+1}\right)^{2}}, \frac{4-z_{n+1}^{2}-|\bar{z}|^{2}}{|\bar{z}|^{2}+\left(2+z_{n+1}\right)^{2}}\right)
$$

where $\left(\bar{z}, z_{n+1}\right) \in \mathbb{R}_{+}^{n+1}$ and $\bar{z}=\left(z_{1}, \ldots, z_{n}\right)$. Note that $\Sigma^{-1}:\left(\mathbb{R}_{+}^{n+1}, g_{E}\right) \rightarrow\left(B^{n+1} \backslash\right.$ $\left.\{S\}, g_{0}\right)$ is a conformal map such that

$$
\left[\left(\frac{4}{\left(2+z_{n+1}\right)^{2}+|\bar{z}|^{2}}\right)^{\frac{n-1}{2}}\right]^{\frac{4}{n-1}} g_{E}=\left(\Sigma^{-1}\right)^{*}\left(g_{0}\right) .
$$

For $u \in C^{\infty}\left(B^{n+1}\right)$, let

$$
v(z)=\left(\frac{4}{\left(2+z_{n+1}\right)^{2}+|\bar{z}|^{2}}\right)^{\frac{n-1}{2}} u\left(\Sigma^{-1}(z)\right) .
$$

It follows from (3.4) that

$$
\left[\left(\frac{4}{\left(2+z_{n+1}\right)^{2}+|\bar{z}|^{2}}\right)^{\frac{n-1}{2}}\right]^{\frac{n+1}{n-1}}\left(\frac{2}{n-1} \frac{\partial}{\partial \nu_{g_{0}}} u+u\right)\left(\Sigma^{-1}(z)\right)=\frac{2}{n-1} \frac{\partial}{\partial \nu_{g_{E}}} v(z) .
$$

This implies that the mean curvatures of $g=u^{\frac{4}{n-1}} g_{0}$ and $\tilde{g}=v^{\frac{4}{n-1}} g_{E}$ are related by

$$
u^{-\frac{n+1}{n-1}}\left(\frac{2}{n-1} \frac{\partial u}{\partial \nu_{g_{0}}}+u\right)=H_{g}\left(\Sigma^{-1}(z)\right)=H_{\tilde{g}}(z)=\frac{2}{n-1} v^{-\frac{n+1}{n-1}} \frac{\partial}{\partial \nu_{g_{E}}} v
$$

Therefore, by (2.5), (2.6) and (3.5), we have

$$
E_{f}[u]=\left(\omega_{n}^{-1} \int_{\partial \mathbb{R}_{+}^{n+1}} H_{\tilde{g}}(z) v(z)^{\frac{2 n}{n-1}} d z\right) /\left(\omega_{n}^{-1} \int_{\partial \mathbb{R}_{+}^{n+1}} f\left(\Sigma^{-1}(z)\right) v(z)^{\frac{2 n}{n-1}} d z\right)^{\frac{n-1}{n}} .
$$


Lemma 3.1. For any point $x_{0} \in S^{n}$ and any positive number $\epsilon>0$, there exists a function $u_{0} \in C^{\infty}\left(S^{n}\right)$ such that

$$
E_{f}\left[u_{0}\right] \leq \frac{1}{f\left(x_{0}\right)^{\frac{n-1}{n}}}+\epsilon .
$$

Moreover, we can choose $u_{0}$ to be invariant under the reflection upon a hyperplane passing through $x_{0}$ and the origin $0 \in R^{n+1}$, and invariant under rotations with axis passing through $x_{0}$ and 0 .

Proof. As the situation is unchanged after a rotation of $S^{n}$, we may assume that $x_{0}=N=(0, \ldots, 0,1)$ the north pole. For $\lambda>0$, we let

$$
v_{0}(z)=\left(\frac{2 \lambda}{|\bar{z}|^{2}+\left(z_{n+1}+\lambda\right)^{2}}\right)^{\frac{n-1}{2}} \text { for } z=\Sigma(x) .
$$

Then $v_{0}$ satisfies the equation:

$$
\begin{aligned}
\Delta v_{0} & =0 \text { in } \mathbb{R}_{+}^{n+1}, \\
\frac{2}{n-1} \frac{\partial v_{0}}{\partial z_{n+1}}+v_{0}^{\frac{n+1}{n-1}} & =0 \text { in } \partial \mathbb{R}_{+}^{n+1},
\end{aligned}
$$

for some $c_{0}>0$. We choose $\lambda$ such that

$$
\int_{\partial \mathbb{R}_{+}^{n+1}} v_{0}(z)^{\frac{2 n}{n-1}} d z=\omega_{n}
$$

If $\tilde{g}=v_{0}(z)^{\frac{4}{n-1}} g_{E}$, then it follows from (3.7) that

$$
\int_{\partial \mathbb{R}_{+}^{n+1}} H_{\tilde{g}}(z) v_{0}(z)^{\frac{2 n}{n-1}} d z=\omega_{n}
$$

We estimate

$$
\begin{aligned}
& \int_{\partial \mathbb{R}_{+}^{n+1}} v_{0}(z)^{\frac{2 n}{n-1}} f\left(\Sigma^{-1}(z)\right) d z \\
& =\int_{\partial \mathbb{R}_{+}^{n+1}} v_{0}(z)^{\frac{2 n}{n-1}}\left[f\left(\Sigma^{-1}(z)\right)-f\left(\Sigma^{-1}(0)\right)\right] d z+f\left(\Sigma^{-1}(0)\right) \int_{\partial \mathbb{R}_{+}^{n+1}} v_{0}(z)^{\frac{2 n}{n-1}} d z \\
& =\int_{\partial \mathbb{R}_{+}^{n+1}} v_{0}(z)^{\frac{2 n}{n-1}}\left[f\left(\Sigma^{-1}(z)\right)-f\left(\Sigma^{-1}(0)\right)\right] d z+f\left(\Sigma^{-1}(0)\right) \int_{\partial \mathbb{R}_{+}^{n+1}} v_{0}(z)^{\frac{2 n}{n-1}} d z \\
& =\int_{B_{\delta}(0)} v_{0}(z)^{\frac{2 n}{n-1}}\left[f\left(\Sigma^{-1}(z)\right)-f\left(\Sigma^{-1}(0)\right)\right] d z \\
& +\int_{\partial \mathbb{R}_{+}^{n+1} \backslash B_{\delta}(0)} v_{0}(z)^{\frac{2 n}{n-1}}\left[f\left(\Sigma^{-1}(z)\right)-f\left(\Sigma^{-1}(0)\right)\right] d z+f\left(\Sigma^{-1}(0)\right) \omega_{n} \\
& =O\left(\epsilon_{1}\right)+O\left(\left[\frac{\lambda}{\delta_{1}}\right]^{n}\right)+f(N) \omega_{n},
\end{aligned}
$$

since

$$
\left|f\left(\Sigma^{-1}(z)\right)-f\left(\Sigma^{-1}(0)\right)\right| \leq \epsilon_{1} \text { whenever } z \in B_{\delta_{1}}(0)
$$

and

$$
\left|v_{0}(z)\right| \leq C\left(\frac{\lambda}{\delta_{1}}\right)^{n} \text { whenever } z \in \partial \mathbb{R}_{+}^{n+1} \backslash B_{\delta_{1}}(0)
$$


for some uniform constant $C$. By first choosing $\delta_{1}>0$ to be small enough so that $\epsilon_{1}>0$ is small and then choosing a small $\lambda>0$ so that $\lambda \cdot \delta_{1}^{-1}$ to be small, we obtain from (3.6), (3.8) and (3.9) that

$$
E_{f}\left[u_{0}\right] \leq \frac{1}{f\left(x_{0}\right)^{\frac{n-1}{n}}}+\epsilon .
$$

Here,

$$
u_{0}(x)=\left(\frac{1}{1+|\bar{z}|^{2}}\right)^{\frac{n-1}{2}} v_{0}(z)=\left(\frac{1}{1+|\bar{z}|^{2}}\right)^{\frac{n-1}{2}}\left(\frac{2 \lambda}{|\bar{z}|^{2}+\lambda^{2}}\right)^{\frac{n-1}{2}} \text { for } z=\Sigma(x),
$$

where $x \in S^{n}$. By (3.1)-(3.3), we have

$$
|\bar{z}|^{2}=\frac{2|\bar{x}|^{2}}{\left|1+x_{n+1}\right|^{2}}=\frac{2\left(1-\left|x_{n+1}\right|^{2}\right)}{\left|1+x_{n+1}\right|^{2}} \text { for } z=\Sigma(x),
$$

where $x \in S^{n}$. That is, $u_{0}$ defined in (3.10) depends only on $x_{n+1}$. One can verify the claimed symmetries directly.

We are ready to prove Theorem 1.2 .

Proof of Theorem 1.2. Without loss of generality, we may assume that

$$
S \in \mathcal{F} \text { and } f(S)=\max _{\mathcal{F}} f
$$

where $S=(0, \ldots, 0,-1) \in S^{n}$ is the south pole. We claim that there is $\delta>0$ such that

$$
f(S) \geq f\left(x_{c}\right)+\delta \text { for any point } x_{c} \in \mathcal{F} \text { with } \Delta_{S^{n}} f\left(x_{c}\right) \leq 0 .
$$

If not, then there exists a sequence of points $x_{c_{i}} \in \mathcal{F}$ such that

$$
\lim _{i \rightarrow \infty} f\left(x_{c_{i}}\right)=f(S)=\max _{\mathcal{F}} f \text { and } \Delta_{S^{n}} f\left(x_{c_{i}}\right)>0 .
$$

By passing to subsequence, we assume that $x_{c_{i}} \rightarrow x_{m} \in \mathcal{F}$ as $i \rightarrow \infty$ such that

$$
f\left(x_{m}\right)=\max _{\mathcal{F}} f \quad \text { and } \quad \Delta_{S^{n}} f\left(x_{m}\right) \geq 0,
$$

which contradicts (1.2). This proves (3.11).

It follows from (3.11) that

$$
\frac{1}{f(S)^{\frac{n-1}{n}}}+\epsilon<\frac{1}{f\left(x_{c}\right)^{\frac{n-1}{n}}} \text { for } x_{c} \in \mathcal{F} \text { with } \Delta_{S^{n}} f\left(x_{c}\right) \leq 0,
$$

where $\epsilon>0$ is a small positive number. Let $u_{0}$ be the positive smooth function constructed in Lemma 3.1. We claim that, with this choice of initial data, Lemma 2.1(i) occurs. Suppose not, Lemma 2.1(ii) occurs. It follows from Lemma 2.3 that $L=1$. Let $Q$ be the blow-up point.

We are going to show that $Q \in \mathcal{F}$. Suppose $Q \notin \mathcal{F}$. Then there exists an isometry $\gamma$ described in Assumption 1.1 or 1.2 such that

$$
\gamma(Q) \neq Q,
$$

which implies that

$$
\max _{\partial^{\prime} B_{r}(\gamma(Q))} u_{k} \leq C \text { for all } k \gg 0
$$


whenever $r$ is small enough, since $\left\{u_{k}\right\}$ is uniformly bounded on any compact subsets of $S^{n} \backslash\{Q\}$ by Lemma 2.2. But Lemma 2.1 implies that

$$
u_{k}(\gamma(x))=u\left(\gamma(x), t_{k}\right)=u\left(x, t_{k}\right)=u_{k}(x) \text { for all } x \in S^{n} .
$$

This together with (3.13) implies that $\max _{\partial^{\prime} B_{r}(Q)} u_{k}$ is uniformly bounded, which contradicts Lemma 2.4(a). This proves that $Q \in \mathcal{F}$.

Hence, by Lemma 2.4(c), $Q \in \mathcal{F}$ and $\Delta_{S^{n}} f(Q) \leq 0$. This together with (3.12) implies that

$$
\frac{1}{f(S)^{\frac{n-1}{n}}}+\epsilon<\frac{1}{f(Q)^{\frac{n-1}{n}}} .
$$

Combining this with Lemma 2.4(d) and Lemma 3.1, we obtain

$$
E_{f}\left[u_{0}\right] \leq \frac{1}{f(S)^{\frac{n-1}{n}}}+\epsilon<\frac{1}{f(Q)^{\frac{n-1}{n}}}=\lim _{k \rightarrow \infty} E_{f}\left[u_{k}\right],
$$

which contradicts (2.7).

Therefore, Lemma 2.1(i) occurs. By Lemma 4.2(i) in [46], $u_{k}$ converges to $u_{\infty}$ along the flow (2.1)-(2.3) such that

$$
\begin{aligned}
\Delta_{g_{0}} u_{\infty} & =0 \quad \text { in } B^{n+1}, \\
\frac{2}{n-1} \frac{\partial u_{\infty}}{\partial \nu_{g_{0}}}+u_{\infty} & =\alpha_{\infty} f u_{\infty}^{\frac{n+1}{n-1}} \text { on } S^{n}
\end{aligned}
$$

for some $\alpha_{\infty}>0$. This proves Theorem 1.2 .

\section{REFERENCES}

1. W. Abdelhedi and H. Chtioui, The prescribed boundary mean curvature problem on the standard n-dimensional ball. Nonlinear Anal. 67 (2007), 668-686.

2. W. Abdelhedi, H. Chtioui, and M. Ould Ahmedou, Conformal metrics with prescribed boundary mean curvature on balls. Ann. Global Anal. Geom. 36 (2009), 327-362.

3. T. Aubin and S. Bismuth, Courbure scalaire prescrite sur les variétés riemanniennes compactes dans le cas négatif. J. Funct. Anal. 143 (1997), 529-541.

4. P. Baird, A. Fardoun, and R. Regbaoui, The evolution of the scalar curvature of a surface to a prescribed function. Ann. Sc. Norm. Super. Pisa Cl. Sci. (5) 3 (2004), 17-38.

5. M. S. Berger, Riemannian structures of prescribed Gaussian curvature for compact 2manifolds. J. Differential Geometry 5 (1971), 325-332.

6. D. Cao and S. Peng, Concentration of solutions for the Yamabe problem on half-spaces. Proc. Roy. Soc. Edinburgh Sect. A 143 (2013), 73-99.

7. S.-Y. A. Chang, M. J. Gursky, and P. C. Yang, The scalar curvature equation on 2 - and 3-spheres. Calc. Var. Partial Differential Equations 1 (1993), 205-229.

8. S.-Y. A. Chang, X. Xu, and P. C. Yang, A perturbation result for prescribing mean curvature. Math. Ann. 310 (1998), 473-496.

9. S.-Y. A. Chang and P. C. Yang, A perturbation result in prescribing scalar curvature on $S^{n}$. Duke Math. J. 64 (1991), 27-69.

10. S.-Y. A. Chang and P. C. Yang, Conformal deformation of metrics on $S^{2}$. J. Differential Geom. 27 (1988), 259-296.

11. S.-Y. A. Chang and P. C. Yang, Prescribing Gaussian curvature on $S^{2}$. Acta Math. 159 (1987), 215-259.

12. X. Chen and P. T. Ho, Conformal curvature flows on compact manifold of negative Yamabe constant. Indiana U. Math. J. 67 (2018), 537-581.

13. X. Chen, P. T. Ho, and L. Sun, Prescribed scalar curvature plus mean curvature flows in compact manifolds with boundary of negative conformal invariant. Ann. Global Anal. Geom. 53 (2018), 121-150.

14. W. Chen and C. Li, Gaussian curvature in the negative case. Proc. Amer. Math. Soc. 131 (2003), 741-744. 
15. W. Chen and C. Li, Prescribing scalar curvature on $S^{n}$. Pacific J. Math. 199 (2001), 61-78.

16. X. Chen and X. Xu, The scalar curvature flow on $S^{n}$-perturbation theorem revisited. Invent. Math. 187 (2012), 395-506.

17. P. Cherrier, Problèmes de Neumann non linéaires sur les variétés riemanniennes. J. Funct. Anal. 57 (1984), 154207.

18. H. Chtioui, M. Ould Ahmedou, and R. Yacoub, Existence and multiplicity results for the prescribed Webster scalar curvature problem on three CR manifolds. J. Geom. Anal. 23 (2013), 878-894.

19. H. Chtioui, M. Ould Ahmedou, and R. Yacoub, Topological methods for the prescribed Webster scalar curvature problem on CR manifolds. Differential Geom. Appl. 28 (2010), 264-281.

20. Z. Djadli, A. Malchiodi, and M. Ould Ahmedou, Prescribing scalar and boundary mean curvature on the three dimensional half sphere. J. Geom. Anal. 13 (2003), 255-289.

21. J. F. Escobar, Conformal metrics with prescribed mean curvature on the boundary. Calc. Var. Partial Differential Equations 4 (1996), 559-592.

22. J. F. Escobar, Uniqueness theorems on conformal deformation of metrics, Sobolev inequalities, and an eigenvalue estimate. Comm. Pure Appl. Math. 43 (1990), 857-883.

23. J. F. Escobar and G. Garcia, Conformal metrics on the ball with zero scalar curvature and prescribed mean curvature on the boundary. J. Funct. Anal. 211 (2004), 71-152.

24. J. F. Escobar and R. Schoen, Conformal metrics with prescribed scalar curvature. Invent. Math. 86 (1986), 243-254.

25. Z. C. Han, Prescribing Gaussian curvature on $S^{2}$. Duke Math. J. 61 (1990), 679-703.

26. Z. C. Han and Y. Y. Li,On the local solvability of the Nirenberg problem on $S^{2}$. Discrete Contin. Dyn. Syst. 28 (2010), 607-615.

27. Z. C. Han and Y. Y. Li, The existence of conformal metrics with constant scalar curvature and constant boundary mean curvature. Comm. Anal. Geom. 8 (2000), 809-869.

28. P. T. Ho, Prescribed curvature flow on surfaces. Indiana Univ. Math. J. 60 (2011), 1517-1542.

29. P. T. Ho, Results related to prescribing pseudo-Hermitian scalar curvature. Int. J. Math. 24 (2013), 29 pp.

30. P. T. Ho, Prescribed Webster scalar curvature on $S^{2 n+1}$ in the presence of reflection or rotation symmetry. Bull. Sci. Math. 140 (2016), 506-518.

31. P. T. Ho, The Webster scalar curvature flow on CR sphere. Part I. Adv. Math. 268 (2015), 758-835.

32. P. T. Ho, The Webster scalar curvature flow on CR sphere. Part II. Adv. Math. 268 (2015), 836-905.

33. J. L. Kazdan and F. W. Warner, Curvature functions for compact 2-manifolds. Ann. of Math. (2) 99 (1974), 14-47.

34. J. L. Kazdan and F. W. Warner, Existence and conformal deformation of metrics with prescribed Gaussian and scalar curvatures. Ann. of Math. (2) 101 (1975), 317-331.

35. M. C. Leung and F. Zhou, Prescribed scalar curvature equation on $S^{n}$ in the presence of reflection or rotation symmetry. Proc. Amer. Math. Soc. 142 (2014), 1607-1619.

36. J. Moser, On a nonlinear problem in differential geometry, Dynamical systems (Proc. Sympos., Univ. Bahia, Salvador, 1971), Academic Press, New York, 1973, 273-280.

37. Q. A. Ngô and H. Zhang, Prescribing Webster scalar curvature on CR manifolds of negative conformal invariants. J. Differential Equations 258 (2015), 4443-4490.

38. T. Ouyang, On the positive solutions of semilinear equations $\Delta u+\lambda u h u^{p}=0$ on the compact manifolds. Trans. Amer. Math. Soc. 331 (1992), 503-527.

39. R. Schoen and D. Zhang, Prescribed scalar curvature on the $n$-sphere. Calc. Var. Partial Differential Equations 4 (1996), 1-25.

40. K. Sharaf, On the boundary mean curvature equation on $\mathbb{B}^{n}$. Bound. Value Probl. (2016), 2016:221.

41. K. Sharaf, H. Alharthy, and S. Altharwi, Conformal transformation of metrics on the $n$-ball. Nonlinear Anal. 95 (2014), 246-262.

42. M. Struwe, A flow approach to Nirenberg's problem. Duke Math. J. 128 (2005), 19-64.

43. J. J. Tang, Solvability of the equation $\Delta_{g} u+\tilde{S} u^{\sigma}=S u$ on manifolds. Proc. Amer. Math. Soc. 121 (1994), 83-92.

44. J. C. Wei and X. Xu, On conformal deformations of metrics on $S^{n}$. J. Funct. Anal. 157 (1998), 292-325. 
45. X. Xu and P. C. Yang, Remarks on prescribing Gauss curvature. Trans. Amer. Math. Soc. 336 (1993), 831-840.

46. $\mathrm{X} . \mathrm{Xu}$ and $\mathrm{H}$. Zhang, Conformal metrics on the unit ball with prescribed mean curvature. Math Ann. 365 (2016), 497-557.

47. H. Zhang, Evolution of curvatures on a surface with boundary to prescribed functions. Manuscripta Math. 149 (2016), 153-170.

Department of Mathematics, Sogang University, Seoul 121-742, Korea

E-mail address: paktungho@yahoo.com.hk, ptho@sogang.ac.kr

Current address: Department of Mathematics, Princeton University, Fine Hall, Washington Road, Princeton NJ 08544 USA

E-mail address: ptho@princeton.edu 